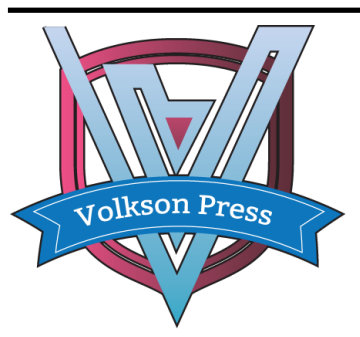

Contents List available at VOLKSON PRESS

New Materials and Intelligent Manufacturing (NMIM)

DOI : http://doi.org/10.26480/icnmim.01.2018.05.07

Journal Homepage: https://topicsonchemeng.org.my/

ISBN: 978-1-948012-12-6

\title{
A PRACTICAL METHOD FOR MEASURING CHARACTERISTIC PARAMETERS OF AUDIO SIGNALS
}

\author{
Zhi Dong Liu, Xue Zhong Ai, Ye Li Yang* \\ Department of control engineering, Jilin Institute of Chemical Technology, No. 45, Chengde street, Longtan District, Jilin, China. \\ *Corresponding Author Email: www.liu847680484@163.com
}

This is an open access article distributed under the Creative Commons Attribution License, which permits unrestricted use, distribution, and reproduction in any medium, provided the original work is properly cited

\section{ARTICLE DETAILS}

Article History:

Received 26 June 2018

Accepted 2 July 2018

Available online 1 August 2018

\section{ABSTRACT}

A practical method for measuring the characteristic parameters of audio signals is introduced. This paper gives the derivation of the formula of mathematical model and expounds the theoretical basis of the measurement of the characteristic parameters of the audio signal. In this paper, the reason of error of measuring frequency and amplitude of the Fourier transform method is analyzed, and the mathematical model for improving measuring accuracy is established by data fitting. By MATLAB simulation, the simulation of the Fourier transform method can be used to measure the characteristic parameters of audio signals.

\section{KEYWORDS}

Voice frequency signal, Sound detection, MATLAB, Simulate the Fourier transform.

\section{INTRODUCTION}

Audio signal processing in mechanical equipment vibration testing, boiler tube leak detection, noise analysis is widely used. The audio signal by the frequency, sampling frequency and the number of quantization bits of digital audio is different, can be divided into four categories: narrowband speech $(300 \sim 3.4 \mathrm{KHz})$, wide band speech $(54 \sim 7 \mathrm{KHz})$, digital audio broadcasting $(20 \sim 15 \mathrm{KHz})$, high fidelity stereo audio signal $(20 \sim 20 \mathrm{KHz})$ $[1,2]$. The sound has its quality characteristics, mainly embodied in three aspects: tone frequency, intensity, and timbre [3]. Human contact sound is generally not a single frequency tone most of it is made of a combination of frequency and amplitude of acoustic wave [4]. We need to find a sound frequency requirements in spectrum analysis.

At present, the main method of audio signal analysis and processing is using digital signal processor (DSP) with digital Fourier transform (FFT) algorithm to achieve the, the utility model has the advantages of fast computing speed, direct access to the results of operations, for laboratory use. The disadvantage is the high cost of circuit, high power consumption, the use of limitations in industrial production is very prominent [5]. Therefore, this paper proposes a method of using analog Fourier transform circuit to measure the audio characteristic parameters. The circuit is low cost, low power consumption, and easy to product.

\section{Basic Principle of Measurement}

When audio signals are processed by Mike and amplifier circuits, they can be equivalent to the following formula:

$$
\mathrm{x}(\mathrm{t})=\mathrm{U}_{\mathrm{m}} \sin \omega_{1} \mathrm{t}
$$

For the upper audio signal, the characteristic parameters are frequency and amplitude.The trigonometric series of square wave signals are expressed in intervals $(0, \mathrm{~T})$ :

$$
f(t)=U_{n}\left[\sin \omega t+\frac{1}{3} \sin 3 \omega t+\frac{1}{5} \sin 5 \omega t \ldots\right]
$$

The square wave signal expansion trigonometric series can be expressed in Figure 1.

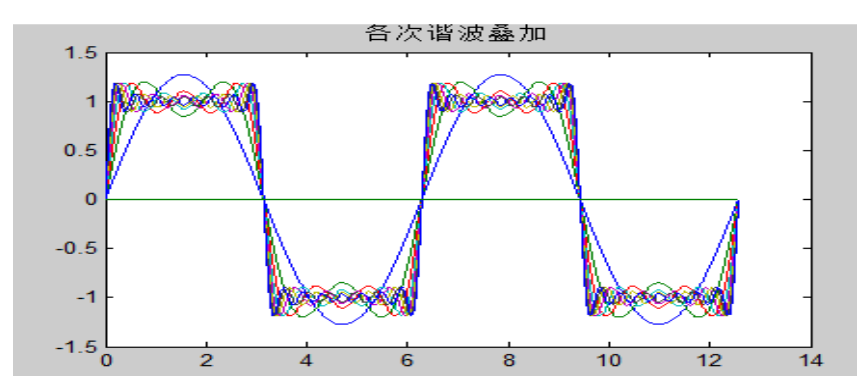

The square wave signal expansion trigonometric series can be expressed in Figure 1.

The audio signal of formula (1) is worked out with square wave signal constructed by formula (2):

$$
\begin{aligned}
& f(t) * x(t)=U_{n} * U_{m}\left[\sin \omega_{1} t * \sin \omega_{2} t+\frac{1}{3} \sin 3 \omega_{2} t * \sin \omega_{1} t+\right. \\
& \left.\frac{1}{5} \sin 5 \omega_{2} t * \sin \omega_{1} t+\cdots+\frac{1}{n} \sin n \omega_{2} t * \sin \omega_{1} t\right]
\end{aligned}
$$

Formulas (3) are computed by item by product and differential operations:

$$
\begin{gathered}
f(t) * x(t)=\frac{U_{n} * U_{m}}{2}\left[\cos \left(\omega_{1}+\omega_{2}\right) t-\cos \left(\omega_{1}-\omega_{2}\right) t+\cdots+\frac{1}{n} \cos \left(\omega_{1}+\right.\right. \\
\left.\left.n \omega_{2}\right) t-\frac{1}{n} \cos \left(\omega_{1}-n \omega_{2}\right) t\right]
\end{gathered}
$$

The low-pass filtering to the formula (4):

$$
\mathrm{F}(\mathrm{t})=\frac{\mathrm{U}_{\mathrm{n}} * \mathrm{U}_{\mathrm{m}}}{2} \cos \left(\omega_{1}-\omega_{2}\right) \mathrm{t}
$$

If the amplitude of square wave signal $U_{n}=1$, the amplitude of $F(t)$ will be half of the amplitude of measured audio signal $x(t)$, and frequency of $\mathrm{F}(\mathrm{t})$ is the difference of square wave signal and measured audio signal $\mathrm{x}(\mathrm{t})$.

\section{STUDY SPECIFIC PROCESSING METHODS OF FREQUENCY AND AMPLITUDE MEASUREMENT}

\subsection{Realization method of frequency measurement}

Theoretically, the square wave infinite subdivision frequency point scanning is adopted, the frequency of $F(t)$ is strictly synchronous, filter output $F(t)$ is direct-current, its voltage can be directly reflected the amplitude of $x(t)$. The frequency of square wave is the frequency of $x(t)$. But taking into account the actual situation of the project, during the 
scanning process, the frequency of measured signal $x(t)$ can be represented:

$$
f_{x(t)}=f_{F(t)}+f_{f(t)}
$$

\subsection{Realization method of amplitude measurement}

A sine wave is a projection of a circular motion in a straight line, as shown in Figure 2, so the amplitude of it can be solved by orthogonal decomposition

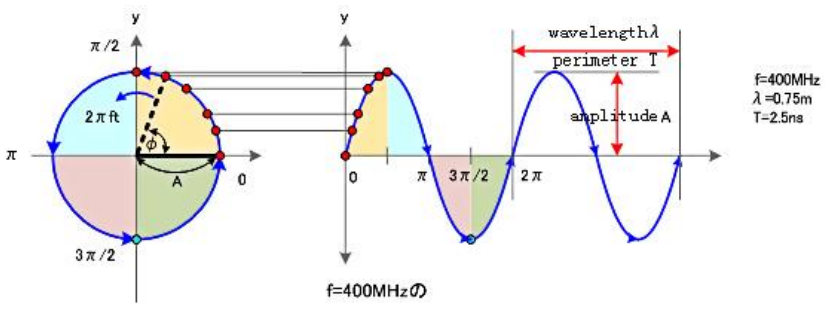

Figure 2: Sine wave generation

Using the phase difference of 90 degrees of the two square wave signals respectively do multiplication and the measured signal, low pass filter is obtained after the phase difference of 90 degrees of two sine wave synchronous sampling of the two-sine wave, can be equivalent to the orthogonal sinusoidal signal decomposition. if synchronous sampling results for a, b. The amplitude of the unknown sine wave can be calculated as $\mathrm{V}_{\mathrm{m}}$.

$$
V_{m}=\sqrt{a^{2}+b^{2}}
$$

\section{VERIFICATION}

\subsection{Single path scan modeling simulation}

In the SIMLINK simulation module of MATLAB, building the model shown in Figure 3. The measured sine wave signal and square wave product are calculated and then output $F(t)$ by four order low-pass filtering. In figure 4 , the measured signal $x(t)$ and the square wave signal $y(t)$ have the same frequency and output $\mathrm{F}(\mathrm{t})$, and the result is only the $\mathrm{DC}$ component. In Figure 5, the measured signal $x(t)$ and square wave signal $y(t)$ frequency differ $20 \mathrm{~Hz}$, and the output $\mathrm{F}(\mathrm{t})$ is $10 \mathrm{~Hz}$ sine wave. From the simulation waveform parameters, it can be seen that the $\mathrm{F}(\mathrm{t})$ agrees with the formula (5) and (6).

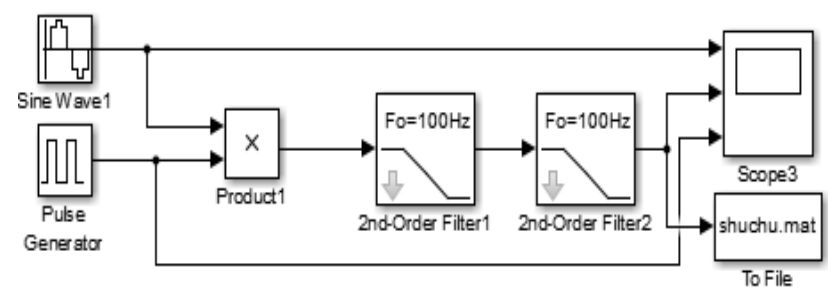

Figure 3: One way scanning simulation model

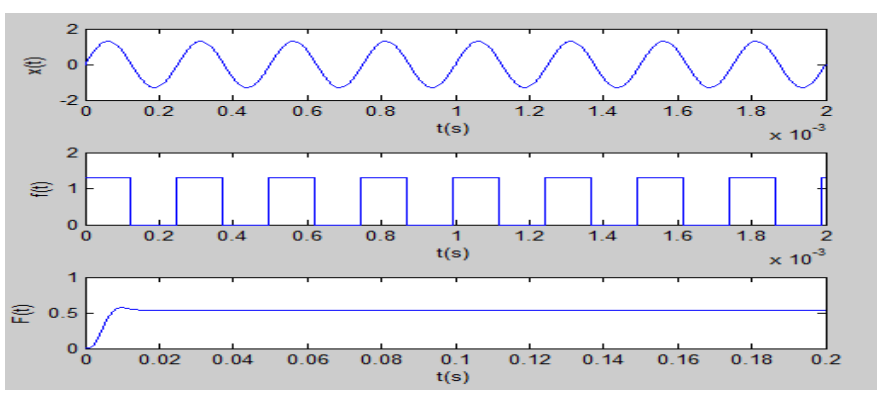

Figure 4: $\mathrm{X}(\mathrm{T})$ and $\mathrm{f}(\mathrm{T})$ have the same frequency and phase

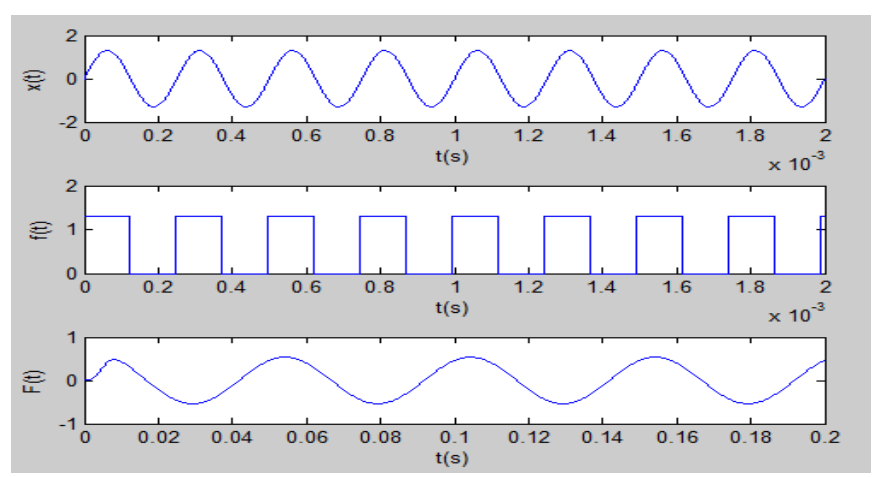

Figure 5: $X(t)$ and $f(t)$ frequency differs $20 \mathrm{~Hz}$

4.2 Double path scanning, orthogonal decomposition, modeling, simulation

In the SIMLINK simulation module of MATLAB, build the model shown in Figure 6. The measured signal is selected as sinusoidal signal, After the product of square wave signal $f_{1}(t) 、 f_{2}(t)$ with 90 phase difference of two degrees, output $F_{1}(t)$ 和 $F_{2}(t)$ after four steps low-pass filter. In theory, $F_{1}(t)$ and $F_{2}(t)$ are same frequency, same amplitude, phase difference of 90 degrees, which is verified in the simulation results of Fig. 7. Synchronous sampling of $F_{1}(t)$ and $F_{2}(t)$ is the $A$ and $B$ in formula (7), the actual amplitude $\mathrm{Vm}$ of the signal can be calculated.

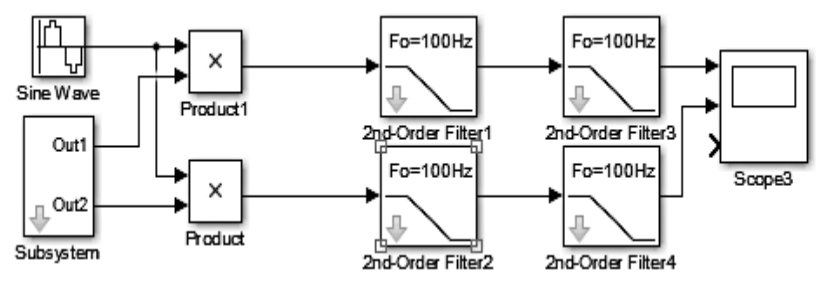

Figure 6: Orthogonal decomposition model of dual path scanning

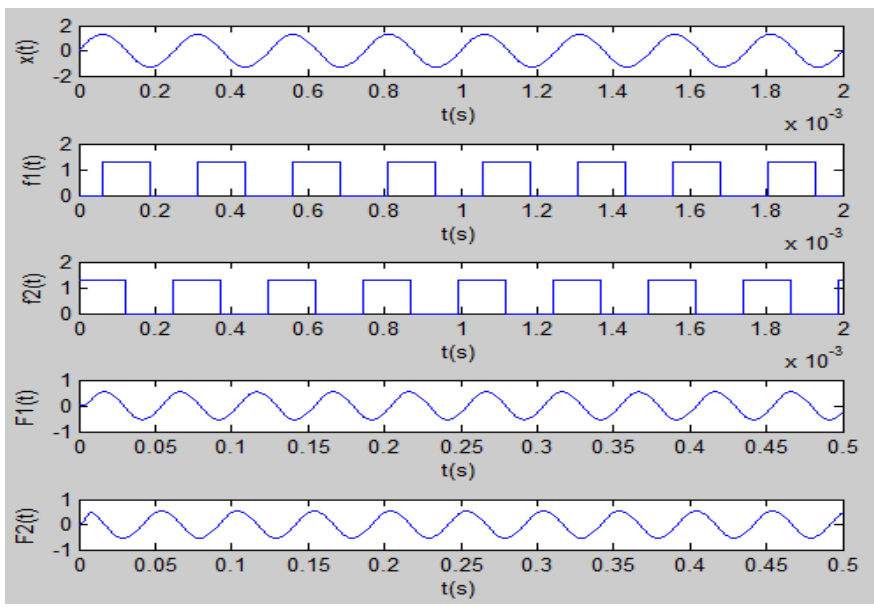

Figure 7: Simulation waveforms of orthogonal decomposition of dual path scanning

\section{AMPLITUDE CORRECTION}

When the signals of same amplitude are scanned by different frequency square wave signals, the amplitude of the output signals is different due to the low-pass filter characteristics, and this phenomenon will affect the measurement accuracy [6]. In order to solve this problem, the measured data are used to fit the function between the frequency difference and the amplitude attenuation coefficient, which is used to correct the measurement error caused by the low pass filter.

Table 1: Frequency and amplitude parameters

\begin{tabular}{|ccccccc|}
\hline $\begin{array}{l}\text { Frequency } \\
\text { difference x }\end{array}$ & 0 & 20 & 40 & 60 & 80 & 100 \\
\hline $\begin{array}{c}\text { Amplitude } \\
\text { correction } \\
\text { factor K }\end{array}$ & 1 & 0.99 & 0.84 & 0.74 & 0.58 & 0.50 \\
\hline
\end{tabular}


Using MATALB, draw the fitting curve shown in figure, as shown in Figure 8:

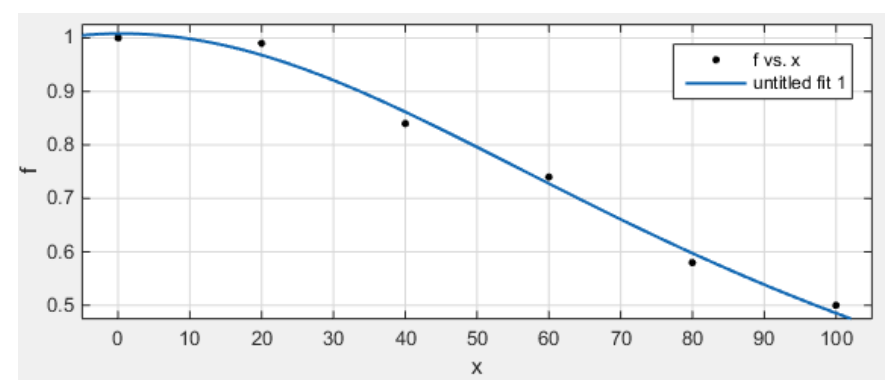

Figure 8: $\mathrm{f}(\mathrm{t})$ simulation function curve

Approximate expression of amplitude correction coefficient $\mathrm{k}(\mathrm{x})$ and frequency difference $x$ [7]:

$$
\mathrm{k}(\mathrm{x})=\frac{9514}{\mathrm{x}^{2}-2.754 * \mathrm{x}+9480}
$$

According to $\left\{\begin{array}{c}V_{1}=V * k(x) \\ V_{2}=V * k(n-x)\end{array}\right.$, among them, $V_{1}, V_{2}$ are the measured amplitudes of the signals to be measured at adjacent known frequency points $f_{1}$ and $f_{2} ; n=f_{2}-f_{1}$ is the sweep stepped value; $V$ is the actual amplitude of the signal to be measured; $X$ is the frequency difference between the measured signal frequency and the sweep frequency point $\mathrm{f}_{1}$; $(n-x)$ is the frequency difference between the measured signal frequency and the sweep point $f_{2}$. Through the above formula, we can calculate the actual amplitude of $\mathrm{V}$ and $\mathrm{X}$, and $\mathrm{V}$ is the measured signal, $\mathrm{f}_{1}+\mathrm{x}$ is the frequency of the signal to be measured.

\section{CONCLUSION}

The characteristic parameters of the audio signal can be measured by the analog Fourier transform, and the accuracy of the signal amplitude can be effectively improved by setting up the actual correction model. This method has low requirement for processor speed and peripheral signal processing circuit and can effectively reduce system cost. This technology has been applied in the leak monitoring system of boiler pressure pipeline, and it has important reference value for solving the problem of signal spectrum analysis and measurement.

\section{REFERENCES}

[1] Yi-heng, Z., Qi-mei, C., Yu-xiang, L. 2008. A Novel TOA Estimation Method with Effective NLOS Error Reduction. The Journal of China Universities of Posts and Telecommunications, 15 (1), 32-37.

[2] Jian, L., Li-kun, W., Yan, Z. 2003. Study on Detection Technique for Pipeline Leakage. Transactions of Tianjin University, 9 (2), 112-114.

[3] Shindoi, T., Hirai, T., Takashima, K. 1999. Plant Equipment Diagnosis by Sound Processing. IEEE, 1020-1026.

[4] Self, R.H. 2003. Jet Noise Prediction Using the Lighthill Acoustic Analogy. Journal of Sound and Vibration, (275), 757-768.

[5] Hileman, J., Thurow, B., Samimy, M. 2004. Development and Evaluation of a 3-D Microphone array to Locate Individual Acoustic Sources in a Highspeed Jet. Journal of Sound and Vibration, (276), 649-669.

[6] Sotiac, P., Music, A.N. 2007. Maximum Like-hood and Cramer-Rao Bound. IEEE Journal of Oceanic Engineering, 1 (2), 720-741.

[7] Charles, H., Knapp, G., Carter, C. 1976. The Generalized Correlation Method for Estimation of Time Delay. IEEE Transactions on Acoustics, Speech, and Signal Processing, 24 (4), 320-327. 